\title{
Auditory evoked phosphenes in optic nerve disease
}

\author{
NGR PAGE, JP BOLGER, MD SANDERS \\ From the Medical Ophthalmology Unit, St Thomas' Hospital, London
}

SUMMARY Five patients with optic neuropathy, four vascular and one demyelinating, are described who each complained of an unusual symptom. Bright flashes of light (phosphenes) occurred in the affected eyes and were evoked by sudden unexpected sounds. Movement of the eye alone did not reproduce the symptom. In all patients the phenomenon was sufficiently prominent to interfere with sleep and was the main complaint of one patient. An anticonvulsant (phenytoin) greatly reduced the frequency and intensity of the phosphene in one patient.

Phosphenes are visual sensations perceived in the absence of visual (luminous) stimuli and may occur in optic nerve disease, but are unusual. Eye movement phosphenes in optic neuritis are now well recognised ${ }^{1}$ and phosphenes induced by sound have been described in three patients with different ocular conditions. ${ }^{2}$ Other positive visual phenomena may also be seen in optic nerve disease. Chromatopsia may be a feature of Leber's optic neuropathy and tabetic optic atrophy and may occur in druginduced toxic optic neuropathy. ${ }^{3}$ We have observed central visual loss in Leber's optic neuropathy due to scotomata described as "dense clouds of brilliant coloured lights". We describe five patients with anterior optic nerve disease with sound or auditory evoked phosphenes and discuss the possible mechanism for this. All the patients presented with rapid unilateral visual loss, optic disc swelling and large sectorial visual field defects.

\section{Case 1}

A 55-year-old man, whilst rubbing his left eye, suddenly became aware that vision in the lower nasal field of the right eye had become blurred. Vision in that eye deteriorated over the next week, with a resultant acuity of $6 / 60$. There was no headache or pain on eye movement but he began to experience disturbing single bright flashes in the right eye initiated by unexpected sounds. The flashes were blue-whiteand discrete, lasting only an instant, and did not contain any formed elements. He was unable to localise the flash within the visual field. This symptom occurred during the day but was most troublesome when he was in a reduced state of wakefulness such as prior to falling asleep. Only unexpected sounds evoked the

Address for reprint requests: NGR Page, St Thomas' Hospital, London SE1 7EH, UK.

Received 8 August 1981

Accepted 29 August 1981 flash; a car door slamming or a cough were the most consistently effective sounds. The onset of a continuous sound might elicit a single discrete flash and there was no "off" phenomenon at the end of the sound. Neither the loudness, nor the direction of the sounds, nor the ear in which it was heard affected the subjective quality of the flash. Anticipated sounds would not evoke a response and nor would blinking or voluntary or involuntary eye movements, however rapid.

Examination at this time did not reveal any abnormalities other than in the right eye. Visual acuity in the right eye was 6/60, N24 and no Ishihara colour plates could be identified. He had a right relative afferent pupil defect. Ocular movements and slit lamp examination were normal. The optic disc was swollen in the superonasal quadrant and the arteries were attenuated. Visual field charting showed an inferonasal sector defect to all isoptres on the Goldmann Chart (fig 1). A diagnosis of ischaemic optic neuropathy was made. Fluorescein angiography showed leakage of fluorescein at the disc with a normal retinal circulation. Visual evoked responses (VER) showed abolished pattern response on the right and the flash VER was extremely subnormal and delayed. The electrooculogram (EOG) and the electroretinogram (ERG) were normal on the right side as was the VER from the left eye. Attempts to evoke a flash by sound or to detect any response in the VER or ERG were unsuccessful. For a period of weeks the symptom persisted causing significant distress and interfered with any form of relaxation. Eventually the frequency of phosphenes reducing to a level allowing normal sleep and ceased after about eight months. His visual acuity has remained at counting fingers and optic atrophy has developed.

\section{Case 2}

A 38-year-old dustman who had been perfectly well, developed a foreign body sensation in his right eye. After two days he awoke with a dark mist over the lower part of the visual field of the right eye. This mist persisted, appearing to fluctuate in intensity over the next week, and has subsequently not changed. On several occasions 

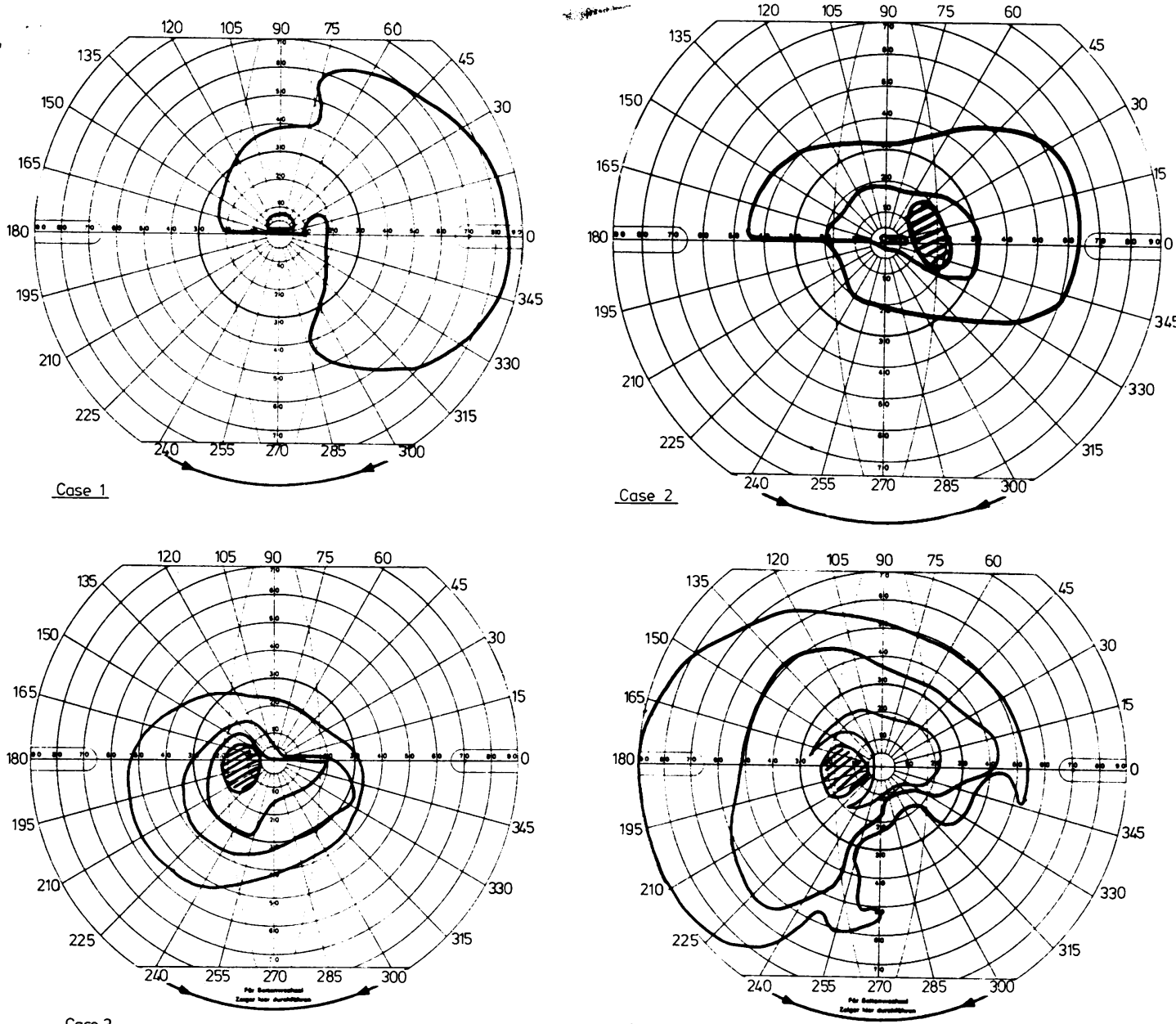

$\underline{\text { Case } 5}$

Fig 1 Visual fields of four affected eyes showing altitudinal and sectorial defects (the visual field could not be charted in one case due to poor acuity).

he had a sharp pain in the right eye on eye movement and in addition had an ache in both temporal regions.

Examination at this time showed visual acuity of $6 / 6$, $\mathrm{N} 5$ in the right eye. Appreciation of light was $60 \%$ compared to the left and there was a right afferent pupil defect. Perimetry showed an inferior altitudinal loss, with generalised depression superiorly in the right eye (fig 1). On fundal examination the optic disc was swollen with a superficial linear peri-papilliary haemorrhage (fig 2). The vessels and retina were normal. Two weeks after onset he began to develop flashes of light similar to the other cases. These occurred at night prior to falling asleep. A loud noise startling him caused a momentary bright white phosphene in the centre of the right eye whereas a quiet sound would cause a dull yellow phosphene. Again, he said this was often caused by his wife talking or moving in bed, or animals or cars outside.
The flash was often caused by the sound of his own coughing. Production of these flashes by voluntary eye or head movements was not possible. The diagnosis of ischaemic optic neuropathy was made. CSF analysis was normal as were investigations for an underlying vascular cause. Fluorescein angiography confirmed disc leakage. The visual evoked response had a low amplitude, poorly defined waveform and the latency was increased. Auditory evoked responses and an EEG were normal, and it was again impossible to show any response to unexpected sounds with these tests, although we were able to provoke phosphenes by sounds whilst he was resting in darkness. A tactile stimulus causing a startle response did not cause a phosphene. He was treated with phenytoin $300 \mathrm{mg}$ daily with a reduction in the frequency and intensity of the phosphenes, such that loud sounds would then only sometimes provoke a dull yellow flash. 

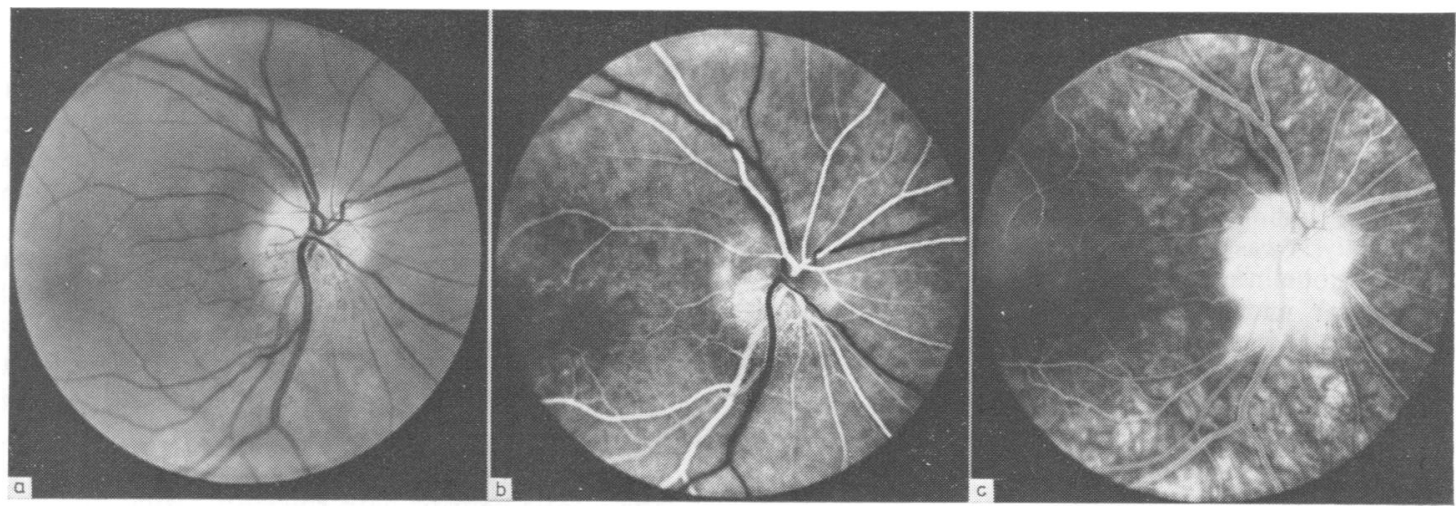

Fig 2 Case 2. Fundus photograph (a) and fluorescein angiograms $(b, c)$ showing a swollen leaking optic disc with dilated capillaries and a superficial haemorrhage.

\section{Case 3}

A 68-year-old man noticed a white-yellow flash of light in his left eye which was elicited by sounds. The patient, a retired optician, observed progress of this symptom with interest. He reported that it occurred only on hearing sudden or unexpected sounds particularly when he was feeling sleepy. Voluntary eye movements could not produce it. The symptoms were remarkably like those of Case 1 in that the discrete flash was always of the same character and produced by sounds such as car doors closing, coughing, footsteps or his wife turning over in bed. Although the symptoms became more disturbing he did not seek advice until he awoke one morning with blurred vision in the left eye. Examination of the eye showed acuity of 6/9, N10. There was an afferent pupil defect and a pale waxy swollen disc with a few intra retinal haemorrhages nearby. The Goldmann field demonstrated upper arcuate loss (fig 1) and examination of the right eye was normal. A diagnosis of left ischaemic optic neuropathy was made. He was otherwise fit with no other abnormalities on examination or haematological or biochemical testing. His vision did not improve and the flashes of light became less frequent over the next four months before disappearing.

\section{Case 4}

A 66-year-old man presented in 1977 with rapid visual loss in the left eye and scalp tenderness. In the past he has been treated for arthritis of the neck and shoulders and, shortly before admission, he developed misty vision and increasing stiffness of the limb girdles.

Examination did not show any clinical evidence of temporal arteritis and the general systems were normal. There were no abnormalities of the right eye but on the left side there was a large central scotoma. Vision was only perception of movement in the lower half and of light in the upper half of the visual field peripherally and the optic disc was pale and swollen. Sedimentation rate was $74 \mathrm{~mm} /$ hour and, although a left temporal artery biopsy was inconclusive, a diagnosis of giant cell arteritis with ischaemic optic neuropathy was made and he was started on corticosteroids. His general health improved but with no change in vision, and he has remained on a small maintenance dose of steroids. A month later he noticed white flashes in the affected eye. These phosphenes were like "a photographic flash" and appeared in the central part of the left eye. He was a light sleeper and the phosphenes occur "in the quiet of night" when he was awake. Any unexpected noise, whether loud or quiet, and not always startling, would immediately evoke a phosphene, and this now occurred several times each night. Again, these phosphenes could not be produced by voluntary or involuntary eye movements or blinking. The auditory evoked phosphenes have continued unchanged for four years and do not now distress him.

\section{Case 5}

A 30-year-old seaman noticed some blurring of vision in the left eye which deteriorated over the next two days and then persisted. Simultaneously, he developed pain in the left eye and orbit extending to the temple, which was constantly present but exacerbated by eye movements. These complaints persisted and after two weeks he began to notice disturbing flashes of light in the left eye associated with sounds, particularly when unexpected. These only occurred in the dark or when his eyes were closed and consisted of a bright flash like "forked lightning crossing the eye". Soft sounds would provoke a dull yellow flash and louder sounds a bright pale blue flash. The phosphene might occur many times daily and occasionally wakes him from sleep. The sound volume necessary for the induction was variable, and unexpected soft sounds, like his wife moving in bed have provoked a flash of light and a "feeling of static". No other positive visual phenomena were experienced and phosphenes could not be elicited by any eye movements or by deliberately making a loud noise. Visual acuity was improving on admission, and treatment had been commenced with steroids.

Examination showed visual acuity in the left eye of $6 / 12+2$ (previously noted at 6/60), N6 with slightly impaired colour vision. The right eye was normal. The pupils were equal with a left relative afferent pupil defect. The left visual field showed an enlarged blind

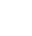


spot with some lower sectorial field loss (fig 1). The disc was pale and slightly elevated with a deep peripapilliary haemorrhage inferonasally. The vitreous was not detached and a resolving macular star was visible.

General examination and in particular the neurological examination was normal. All investigations were normal including tests for syphilis and other infections. CSF analysis was normal and no evidence of intrathecal immunoglobulin synthesis was detected. Vision continued to improve and a clinical diagnosis of optic neuritis was made. After seven months the phosphenes were still occurring frequently and were unchanged in character. The recent onset of episodic hemianaesthesia, suggested the development of multiple sclerosis.

\section{Discussion}

Five patients are described with unilateral rapid visual loss and positive visual phenomena in the affected eyes. Their symptoms are summarised in the table. These included flashes of light which could be white, blue or yellow, but most frequently a bright white flash with a bluish tinge. The flash occurred centrally in one (Case 2), passed temporally to nasally in one (Case 5) and could not be localised in the remaining three. In all cases the flashes were regularly perceived whilst falling asleep and the symptom occurred in daylight in only one patient (Case 1); the others would only have the symptom in the dark or with their eyes closed and this occurred occasionally in all patients when they were fully alert. Eye movements never provoked phosphenes except initially in Case 2 and no patient admitted to any other positive visual phenomena. Only unexpected sounds, particularly those startling them, were effective. All of the patients agreed that sounds from another room or outside the building were the most effective.

None of the patients had any previous illness and no systemic abnormalities were found during investigation of their optic nerve disease other than giant cell arteritis in one (Case 4). The posterior segment was closely examined by indirect ophthalmoscopy and three-mirror fundoscopy in all patients, looking for vitreous detachment but this was never seen. Case 5 had a macular star. Non-arteritic ischaemic optic neuropathy was the clinical diagnosis in Cases 1, 2 and 3. They were all middle-aged men with relatively rapid onset of unilateral visual loss, with swollen discs that became pale, without recovery of vision. Their clinical profiles accord well with the description by Eagling, Sanders and Miller. ${ }^{4}$ Case 2 was unusual in having pain on eye movement during the first week. Giant cell arteritis caused ischaemic optic neuropathy in a further case (Case 4).

In Case 5 the clinical picture was that of demyelinating optic neuritis with subacute visual loss and some subsequent recovery attended by typical pain on eye movement. There was no other evidence of multiple sclerosis at presentation, but his subsequent progress has been suggestive of this diagnosis. The pattern of visual field loss and the appearance of the optic disc was, however, very similar to that of the patients with ischaemic optic neuropathy.

In addition to the examination of visual evoked responses in all patients, electroretinography was performed in two (Cases 1 and 2). In these, various auditory stimuli were provided during both types of recordings but, since it was not possible to provoke phosphenes by expected sounds and since responses must be averaged, no result was obtained. Similarly, a sleep EEG was recorded in one (Case 2) and phosphenes were provoked by unexpected sounds but no abnormality was obtained other than an arousal pattern. Tactile stimuli which did not evoke phosphenes caused a similar arousal pattern.

Phosphenes or photopsia are well described and documented ophthalmic symptoms. They usually consist of a flash similar to lightning or a shower of sparks. When provoked in normal people by rubbing or pressing on the globe of the eye bright sparks, sometimes surrounded by a halo, can be seen. ${ }^{5}$ Phosphenes may be reported by patients with

Table Summary of symptoms. (In all patients phosphenes occur in the affected eye and are most likely when falling asleep and startled by unexpected sounds)

\begin{tabular}{lllll}
\hline & Diagnosis & Onset of phosphenes & Nature \\
\hline Case 1 & $55 / \mathrm{M}$ & ION & After visual loss & $\begin{array}{l}\text { Blue/white, } \\
\text { not localised, } \\
\text { in darkness or light } \\
\text { Blue/white or dull yellow, } \\
\text { central, } \\
\text { in darkness } \\
\text { White flash in darkness, } \\
\text { not localised } \\
\text { White flash in darkness, } \\
\text { not localised } \\
\text { White or dull yellow } \\
\text { in darkness, } \\
\text { passing temporally to nasally }\end{array}$ \\
Case 4 & $38 / \mathrm{M}$ & ION & After & Before \\
\hline
\end{tabular}


various disease processes affecting the posterior segment, usually vitreous detachment, a common benign condition. The detached gel tugs on the retinal nerve cells and causes a subjective sensation of light. Phosphenes are generated by an identical mechanism in retinal detachment and the amorphous flash of showers of white sparks are usually provoked by eye movement. ${ }^{6}$ Micro-emboli passing through the retinal arterioles may cause phosphenes. These usually present as a bright golden light that slowly follows an arcing course across the upper or lower part of the visual field of the affected eye.

Davis et $a l^{1}$ reported nine patients with optic neuritis or multiple sclerosis who exhibited phosphenes in the affected eye on voluntary movement. They reported the vitreous to be clear of floaters but not whether it was attached. They felt this phenomenon was probably due to mechanical movement of a demyelinated nerve evoking discharge potentials and was an analogous to the Lhermitte symptom. They cited the experimental evidence that unmyelinated lobster axons discharged more readily than myelinated axons on mechanical stimulation. ${ }^{7}$

Loud or unexpected sound may cause eye movement in the direction of the stimulus. Such movements, in susceptible individuals, may cause generation of phosphenes by agitation of a detached vitreous gel or movement of demyelinated optic nerve. However, by the same token, rapid voluntary eye or head movement should be effective and phosphenes could not be evoked by such movements in our patients.

Positive visual phenomena arising from the striate cortex tend to have some formed content. Irritation of the visual association areas or temporal lobes may cause formed hallucinations. Phosphenes from the striate cortex consist of less formed elements often with an angular form but nevertheless are not amorphous as retinal phosphenes. Thus, in the preheadache phase of classical migraine, scintillations around the negative scotoma and the mosaic pattern seen reflect the organisation of the columnar cortex units. Characteristically occipital phosphenes are not evanescent but endure and since they usually arise on one side of the brain they are perceived in one hemifield and cannot be localised to one eye. Our patients symptoms do not correspond with these phosphenes.

It seems clear that the sounds required to evoke phosphenes in our patients caused a startle or alerting response. It is at the lateral geniculate nucleus (LGN) that the visual pathway first converges with other central nervous system pathways and it is here that visual information en route to the cortex may be modified by non-retinal influences. This has been shown experimentally on normal animals. Suzuki and Taira ${ }^{8}$ showed that in cats stimulation of the reticular activating system (RAS) affected transmission in the LGN. Facilitatory effects on the responsiveness of striate cortex units to liminal optic tract stimuli were obtainable by stimulation of a limited region of the mesencephalon. Marchiafava and Pompeiano, ${ }^{9}$ by repetitive stimulation of the VIIIth nerve, increased the excitability of the intrageniculate optic tract. Prolonged changes in the tonic activity of the LGN and visual radiations can be induced by tactile and vibration stimuli.10 Later Skrebitsky ${ }^{11}$ found that units in the visual cortex reacted to acoustic stimuli and while the responses were non-specific, he also found that sensory arousal produced faciliation of both spontaneous and photically evoked activity in the LGN. In a review of the non-visual influences on the LGN, Mcllwain ${ }^{12}$ suggested that these influences operate a gate or gain control function during different levels of consciousness and sleep, tuning the quality of the visual resolution to the needs of the animal in its particular state of arousal. These experiments may explain auditory evoked phosphenes.

The gating mechanism in the LGN, receiving an abnormal input from a damaged optic nerve, may function abnormally such that other inputs may pass through abnormal LGN gates and be perceived as visual sensations. These inputs could be direct auditory stimuli or a RAS arousal response. However, this seems improbable because such an input would be perceived in one or both cortices and could not be uniocular. Therefore, it may be that subliminal potentials are generated in the damaged optic nerve or peripapilliary retina and these may be enhanced by reticular system stimulation so that their passage through the LGN to perception is facilitated. Cortical rather than collicular perception seems more likely.

Lessell and Cohen ${ }^{2}$ suggest that under conditions of altered excitability produced by visual deafferentation and in the early stages of sleep, cells capable of responding to both visual and auditory stimuli become hyper-responsive. They postulate that, perhaps by ephaptic transmission between areas of different pathways in sites where the auditory and visual pathways are closely associated, an auditory stimulus may provoke a phosphene which may be related to the direction of the sound. Their patients differed from ours in that theirs were able to discern a spatial relationship between the flashes and the sound stimuli although it is not clear whether all their patients could localise the flash to a particular eye. In our patients the flashes were not related to the direction from which the sound came and they could localise them to the diseased eye. All our patients were certain of this, although objective proof was impossible; in one case the phosphene 
was seen passing horizontally across the visual field and another saw it in the centre of vision. If the flashes appear in one eye only and are perceived in the striate cortex then both cerebral hemispheres are probably involved and it seems unlikely that simultaneous ephaptic transmission could occur in two separate sites. For this reason we think that these auditory evoked phosphenes are generated in the retina or optic nerve and must be transmitted bilaterally through the lateral geniculate nuclei.

The exact mechanism remains speculative. Electrophysiological tests on our patients have not been able to document auditory evoked phosphenes. We suggest however that they are manifestations of brain stem influences, occurring as part of the arousal mechanism on the gating system of the lateral geniculate nuclei and thus the visual pathways.

The authors express their thanks to Dr R Ross Russell and $\mathrm{Dr} \mathrm{H}$ Ikeda for helpful criticism and to $\mathrm{Mr} \mathrm{R}$ Sennhenn for photography and Miss G Braganza for secretarial assistance.

\section{References}

${ }^{1}$ Davis FA, Bergen D, Schauf C, McDonald WI, Deutsch W. Movement Phosphenes in optic neuritis: A new clinical sign. Neurology (Minneap) 1967;26:
1100-4.

${ }^{2}$ Lessell S, Cohen MM. Phosphenes induced by sound. Neurology (Minneap) 1979;29:1524-7.

${ }^{3}$ Walsh FB, Hoyt WF. Clinical Neuro-ophthalmology. Baltimore: Williams and Wilkins, 1969.

${ }^{4}$ Eagling EM, Sanders MD, Miller SJH. Ischaemic Papillopathy. Br J Ophthalmol 1974;58:940-1008.

${ }^{5}$ Moore RF. Subjective lightening streaks. Br J Ophthalmol $1947 ; 31: 46-50$.

${ }^{6}$ Duke Elder S, Dobree JM. Diseases of the Retina in: Duke Elder; System of Ophthalmology. London: Henry Kimpton, 1967.

${ }^{7}$ Julian JF, Goldman DE. The Effects of Mechanical Stimulation on some electrical axons. J Gen Physiol 1962;46:297-313.

${ }^{8}$ Suzuki H, Taira N. Effect of reticular stimulation upon synaptic transmission in cat's Lateral Geniculate Body. Jpn J Physiol 1961 ;11:641-55.

${ }^{9}$ Marchiafava PL, Pompeiano O. Excitability Changes of the Intra-Geniculate Optic Tract Fibres Produced by Electrical Stimulation of the Vestibular System. Pfluegers Arch 1966;290:275-8.

${ }^{10}$ Melzach R, Konrad K, Dubrovsky B. Prolonges changes in Visual System Acuity Produced by Somatic Stimulation. Exp Neurol 1968;20:443-59.

${ }^{11}$ Skrebitsky VG. Non-specific Influences on Neuronal Firing in the Central Visual Pathways. Exp Brain Res 1969;9:269-83.

12 McIlwain JT. Non-retinal influences on the lateral geniculate nucleus. Invest Ophthalmol 1972;11,5: 311-22. 